\title{
Оптическое поглощение, связанное с межзонными и межподзонными переходами электронов в теллуриде висмута
}

\author{
(C) А.Н. Вейс ${ }^{1}$, Л.Н. Лукьянова ${ }^{2}$, О.А. Усов ${ }^{2}$ \\ ${ }^{1}$ Санкт-Петербургский политехнический университет Петра Великого, \\ 195251 Санкт-Петербург, Россия \\ ${ }^{2}$ Физико-технический институт им. А.Ф. Иоффе Российской академии наук, \\ 194021 Санкт-Петербург, Россия \\ E-mail: alnveis@mail.ru
}

Поступила в Редакцию 20 октября 2021 г.

В окончательной редакции 25 октября 2021 г.

Принята к публикации 25 октября 2021 г.

При температурах 205 и $300 \mathrm{~K}$ исследованы спектральные зависимости коэффициента поглощения $\alpha$ в субмикронных образцах топологического изолятора $n-\mathrm{Bi}_{2} \mathrm{Te}_{3}+\mathrm{CdBr}_{2}$, обладающих при высоких значениях коэффициента Зеебека оптимальными термоэлектрическими свойствами при температурах ниже комнатной. Выявлены и проанализированы составляющие спектров оптического поглощения, связанные с межзонными и межподзонными переходами электронов. Установлено, что в теллуриде висмута при понижении температуры оптические переходы электронов на пороге межзонного поглощения остаются прямыми и разрешенными. Оценены величины энергетических зазоров между абсолютными экстремумами зоны проводимости и валентной зоны, а также между основной и дополнительной подзонами зоны проводимости. Показано, что скорости их изменения с температурой противоположны по знаку. Построена энергетическая схема $\mathrm{Bi}_{2} \mathrm{Te}_{3}$, отвечающая экспериментальным данным.

Ключевые слова: теллурид висмута, топологический изолятор, оптическое поглощение, межзонные и межподзонные переходы, энергетическая зонная схема.

DOI: $10.21883 /$ FTP.2022.02.51956.29

\section{1. Введение}

Теллурид висмута, который принадлежит к группе слоистых полупроводников $\mathrm{A}_{2}^{\mathrm{V}} \mathrm{B}_{2}^{\mathrm{VI}}$, относится к топологическим изоляторам $[1,2]$. Соединение является базовым термоэлектрическим материалом и до сих пор широко используется для создания термоэлектрических устройств, обладающих высокой термоэлектрической эффективностью в области рабочих температур $120-500 \mathrm{~K}[3,4]$. Топологические явления, обнаруженные в термоэлектриках на основе халькогенидов висмута и сурьмы, расширяют возможности практического использования этих материалов в оптике [5], магнетооптике [6], оптоэлектронике [7], спинтронике [8], и термоэлектричестве [9-11].

Несмотря на имеющиеся результаты, энергетические спектры слоистых соединений $\mathrm{A}_{2}^{\mathrm{V}} \mathrm{B}_{2}^{\mathrm{VI}}$, в том числе и теллурида висмута, до сих пор исследованы недостаточно, что затрудняет учет влияния зонной структуры на термоэлектрические свойства. Прогресс в исследовании особенностей зонных спектров слоистых полупроводников $\mathrm{A}_{2}^{\mathrm{V}} \mathrm{B}_{2}^{\mathrm{VI}}$ был достигнут лишь недавно, благодаря более широкому использованию оптических методов для изучения их физических свойств $[5,6,12]$. В этом плане особое значение имеет исследование спектров оптического поглощения, позволяющее получать обширную и нетривиальную информацию о величинах зонных параметров изучаемых соединений и их зависимостей от концентрации свободных носителей заряда и температуры. В частности, исследования спектров коэффициента поглощения $\alpha$, выполненные авторами работы [12], позволили получить независимое доказательство существования дополнительного экстремума в зоне проводимости теллурида висмута и при комнатной температуре определить величину энергетической щели $\Delta E_{c}$ между ним и основным экстремумом электронной зоны.

В настоящей работе продолжены исследования, начатые авторами [12]. Цель выполненных экспериментов состояла в том, чтобы выявить в спектрах коэффициента поглощения области, связанные с межзонными и межподзонными переходами электронов, определить тип межзонных переходов электронов на пороге фундаментального поглощения и оценить скорости температурного изменения энергетического зазора $\Delta E_{c}$ между основной и дополнительной подзонами зоны проводимости и ширины запрещенной зоны $E_{g 0}$ в теллуриде висмута.

\section{2. Объект исследования и методика измерений}

Исследованный в работе монокристалл $n-\mathrm{Bi}_{2} \mathrm{Te}_{3}+\mathrm{CdBr}_{2}$ был выращен методом направленной кристаллизации. Тонкие образцы, необходимые для оптических исследований, были приготовлены методом скола монокристаллических зерен вдоль межслоевой поверхности Ван-дер-Ваальса (0001). Исследованные 
образцы были оптимизированы для области температур ниже комнатной при величине коэффициента Зеебека $S=-275 \mathrm{мкB/K}$. Толщины образцов были определены исходя из положения экстремумов интерференционных полос, наблюдаемых в спектрах их прозрачности, по шкале энергий. Отметим, что исследования коэффициентов отражения и поглощения, выполненные в различных сколах изучаемого кристалла, показали, что в нем существуют слоевые неоднородности в пространственном распределении концентрации свободных носителей заряда. Величины концентрации свободных электронов $n_{e}$ в трех сколах, определенные исходя из частоты плазменных колебаний свободных носителей заряда, варьировались в пределах $(2.8-4.2) \cdot 10^{18} \mathrm{~cm}^{-3}$. В частности, в тонком слое толщиной $d=(3.19 \pm 0.07)$ мкм, результаты исследования которого обсуждаются в настоящей работе, она оказалась равной $3.7 \cdot 10^{18} \mathrm{~cm}^{-3}$ (здесь и далее величины доверительных интервалов определены при надежности $s=0.95)$. Попутно были определены величины высокочастотной диэлектрической проницаемости $\varepsilon_{\infty}$ в исследованном кристалле, которые оказалась равными $(92 \pm 7)$ и $(85.5 \pm 6.5)$ при температурах 300 и $205 \mathrm{~K}$ соответственно.

В настоящей работе особое внимание было уделено обработке интерференционных спектров, полученных методом оптического поглощения. Прежде всего интерференционные спектры, наблюдаемые в исследованном образце, были редуцированы к нулевой щели монохроматора методом Брейсвелла. Затем, исходя из значений прозрачности в максимумах редуцированных спектров $t_{\max }\left(\lambda_{\max }\right)$, были определены величины $\alpha d(\alpha-$ коэффициент поглощения) при длинах волн $\lambda_{\max }$, соответствующих максимумам в интерференционных спектрах в соответствии с работой [13]. На основании полученных таким образом значений $\alpha d$ были определены величины прозрачности исследованного образца $t\left(\lambda_{\max }\right)$, которые наблюдались бы в нем при $\lambda=\lambda_{\max }$ в отсутствие интерференции. Полученные значения $t\left(\lambda_{\max }\right)$ были соединены между собой плавной кривой. Это позволило определить $\alpha$ в точках $\lambda_{i}$, лежащих в промежутках между интерференционными максимумами.

\section{3. Экспериментальные результаты и обсуждение}

Экспериментальные данные, полученные авторами, представлены на рис. 1 кривыми 1 и 2 . Видно, что при понижении температуры никаких новых особенностей в спектре коэффициента поглощения изучаемого кристалла не появляется. В коротковолновой области спектров $\alpha(h v)$ при обеих температурах наблюдается край фундаментальной полосы, в длинноволновой поглощение свободными электронами, отвечающее степенной зависимости

$$
\alpha_{f c} \propto(h v)^{-l}
$$

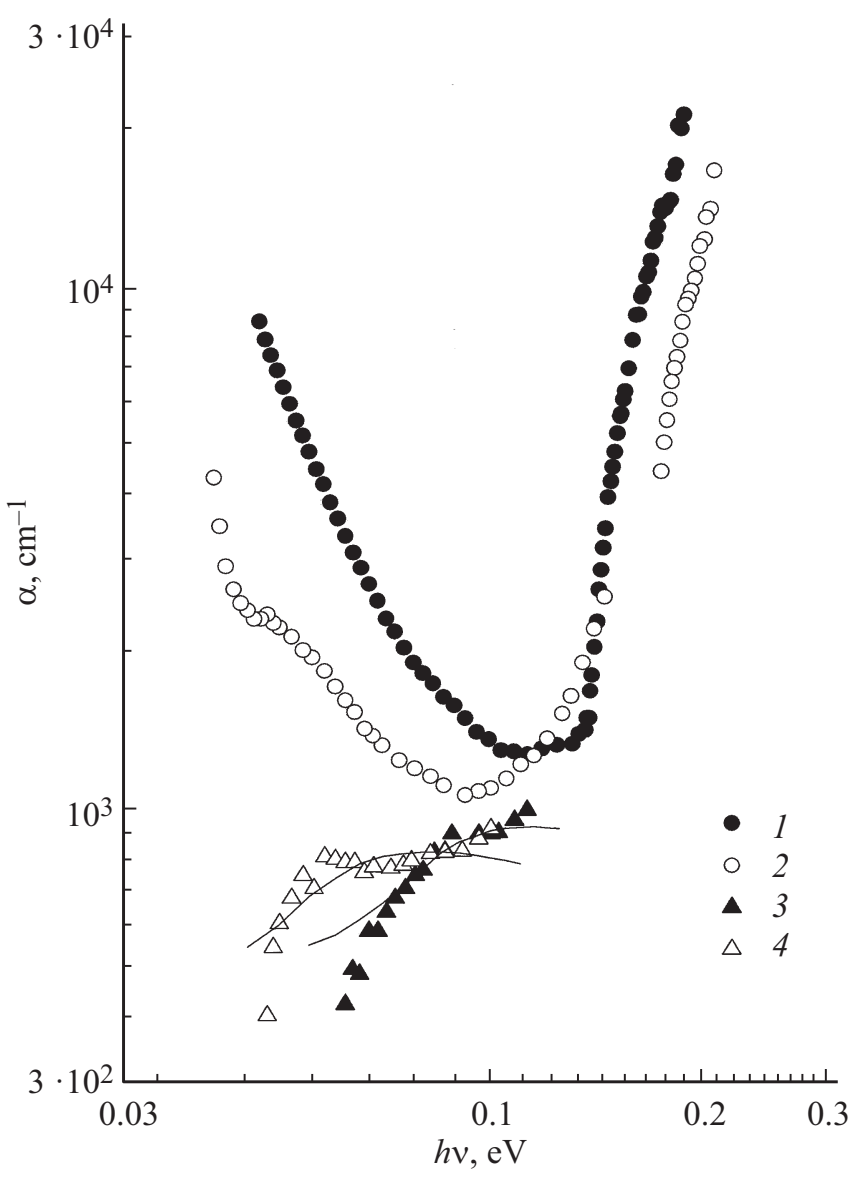

Рис. 1. Спектральные зависимости $\alpha(h v) \quad(1,2)$ и $\alpha_{a d d}(h v)(3,4)$ в $n-\mathrm{Bi}_{2} \mathrm{Te}_{3}$ при температурах $T, \mathrm{~K}: 1,3-$ $300,2,4$ - 205. Точки - эксперимент, линии - результаты расчета, выполненного в соответствии с работой [14], энергетический зазор между основной и дополнительной подзонами зоны проводимости $\Delta E_{c}$, эВ: $3-0.09,4-0.062$.

Там же, в длинноволновой области спектра $\alpha(h v)$, присутствуют полосы дополнительного поглощения $\alpha_{a d d}$, связанные с оптическими переходами электронов из основной в дополнительную подзону зоны проводимости. При комнатной температуре поглощение свободными носителями заряда велико, поэтому полоса $\alpha_{a d d}$ малозаметна. Об ее присутствии в спектре поглощения свидетельствует лишь один факт - излом зависимости $\alpha(h v)$, наблюдаемый при $h v \sim 0.06$ эВ. При температуре $205 \mathrm{~K}$ величины коэффициента поглощения свободными электронами уменьшаются, поэтому полоса $\alpha_{a d d}$ в спектре поглощения становится отчетливо видной. Сопоставление данных, полученных при двух температурах, между собой показывает, что при понижении температуры точка излома зависимости $\alpha(h v)$ смещается в длинноволновую область оптического спектра. Эта особенность уже на этапе качественного анализа полученных данных позволяет сделать заключение о том, что знак скорости температурного изменения величины энергетического зазора между подзонами зоны проводимости $d\left(\Delta E_{c}\right) / d T$ 
в теллуриде висмута положителен. Напротив, сдвиг края фундаментальной полосы в коротковолновую область, наблюдаемый при понижении температуры, указывает на то, что в $\mathrm{Bi}_{2} \mathrm{Te}_{3}$ величина $d\left(E_{g 0}\right) / d T<0$.

\section{4. Параметры энергетического спектра теллурида висмута}

Выделение полос дополнительного поглощения $\alpha_{a d d}$ из экспериментальных спектров, необходимое для количественного анализа их спектральных зависимостей, было выполнено посредством вычитания поглощения свободными электронами, экстраполированного в коротковолновую область по закону (1). При этом были проварьированы значения параметра $l$ в формуле (1) в пределах, допускаемых разбросом экспериментальных точек. Этот прием позволил при обеих температурах получить несколько экспериментальных зависимостей $\alpha_{a d d}(h v)$ (при $300 \mathrm{~K}$ - четыре, при $205 \mathrm{~K}$ - шесть), каждая из которых была проанализирована отдельно.

Определение величин $\Delta E_{c}$ было осуществлено при помощи расчета спектров $\alpha_{a d d}(h v)$, выполненного в рамках теории Хаги и Кимуры [14] для оптических непрямых межподзонных переходов $I$-типа.

Значения $\left(\Delta E_{c}\right)_{i}$, полученные при каждой из температур при помощи расчета спектров $\alpha_{a d d}(h v)$, были усреднены и представлены на рис. 2. Согласие между экспериментальными значениями $\alpha_{a d d}$ и результатами расчета спектров $\alpha_{a d d}(h v)$ проиллюстрировано данными, показанными на рис. 1 (кривые 3 и 4). Отметим, что на рис. 1 приведены только те из полученных экспериментальных результатов, анализ которых позволил получить величины $\left(\Delta E_{c}\right)_{i}$, наиболее близкие к их средним значениям.

На рис. 2 также представлена величина энергетического зазора $\Delta E_{c}$, полученная Кёллером [15] при исследовании осцилляций Шубникова-де Гааза. Видно, что все значения параметра $\Delta E_{c}$, представленные на рис. 2, могут быть объединены линейной зависимостью, обладающей наклоном $d\left(\Delta E_{c}\right) / d T$, лежащим в пределах $(1.7-2.3) \cdot 10^{-4}{ }_{\ni B / K}$.

Для того чтобы определить скорость температурного изменения величины энергетического зазора между абсолютными экстремумами электронной и дырочной зон теллурида висмута $d\left(E_{g 0}\right) / d T$, был выполнен анализ частотных зависимостей коэффициента поглощения $\alpha_{\text {fund }}$ в области края фундаментальной полосы. Для этого составляющие $\alpha_{\text {fund }}$, связанные с межзонными оптическими переходами, были выделены из экспериментальных спектров $\alpha(h v)$ посредством вычитания составляющих $\alpha_{a d d}$ и $\alpha_{f c}$, связанных с межподзонными переходами электронов и поглощением свободными носителями заряда соответственно. Оказалось, что в исследованном кристалле прямые межзонные переходы становятся хорошо заметными при значениях $\alpha_{\text {fund }}$, превышающих $6000-8000 \mathrm{~cm}^{-1}$. Это позволило построить зависимости

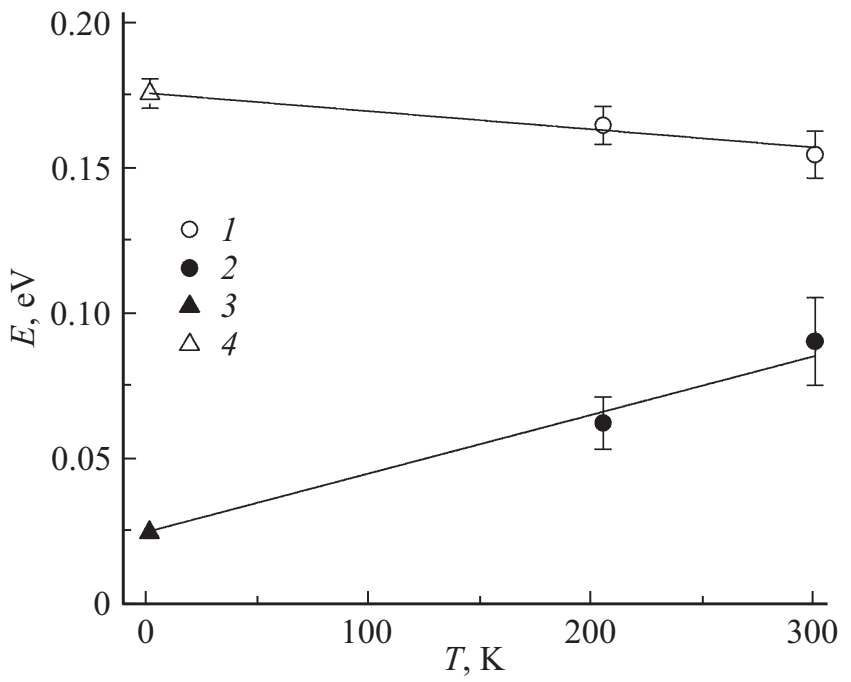

Рис. 2. Зависимости величин энергетической щели между абсолютными экстремумами электронной и дырочной $30 \mathrm{H}$ $E_{g 0}$ (кривая 1) и энергетического зазора между основным и дополнительным экстремумами зоны проводимости $\Delta E_{c}$ в $\mathrm{Bi}_{2} \mathrm{Te}_{3}$ (кривая 2) от температуры. 1,2 - наши данные, 3 данные работы [15], 4 - данные работы [6].

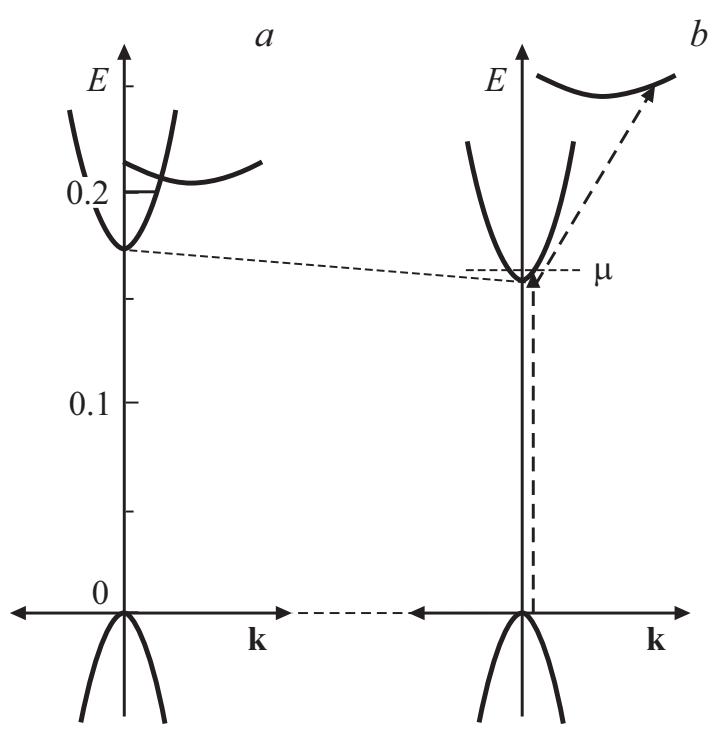

Рис. 3. Энергетическая схема $\mathrm{Bi}_{2} \mathrm{Te}_{3} . T, \mathrm{~K}: a-0, b-$ 300. Пунктирными стрелками показаны оптические переходы электронов.

$\alpha_{\text {fund }}^{2}(h v)$, и по их отсечке на оси абсцисс оценить оптическую ширину запрещенной зоны $E_{\text {орt, }}$ превышающую $E_{g 0}$ вследствие наличия в зоне проводимости исследуемого кристалла газа свободных электронов. При температурах 300 и $205 \mathrm{~K}$ она оказалась равной $(0.166 \pm 0.004)$ и $(0.189 \pm 0.005)$ эВ соответственно. И лишь затем, по методике, развитой в работе [16], были найдены значения $E_{g 0}$. Они представлены на рис. 2. Видно, что в $\mathrm{Bi}_{2} \mathrm{Te}_{3}$ при увеличении температуры запрещенная щель между абсолютными экстремумами 
электронной и дырочной зон уменьшается. На этом же рисунке представлен экспериментальный результат, полученный авторами работы [6] при $T=2 \mathrm{~K}$ методом магнетопоглощения в полях до 34 Тл. Указанный результат также может быть объединен с данными настоящей работы линейной зависимостью с наклоном, абсолютная величина которого вчетверо меньше значения $d\left(\Delta E_{c}\right) / d T$.

Энергетическая схема теллурида висмута, отвечающая полученным в работе экспериментальным данным, показана на рис. 3.

\section{5. Заключение}

Таким образом, данные, полученные в настоящей работе, позволяют считать, что в интервале температур 0-300 K теллурид висмута является прямозонным полупроводником, в котором оптические переходы на пороге фундаментального поглощения - прямые, разрешенные. При возрастании температуры ширина запрещенной щели в $\mathrm{Bi}_{2} \mathrm{Te}_{3}$ уменьшается со скоростью $d\left(E_{g 0}\right) / d T \sim-0.5 \cdot 10^{-4}$ эВ/K.

Показано, что зона проводимости теллурида висмута имеет сложное строение и состоит из двух подзон, разделенных непрямым энергетическим зазором $\Delta E_{c}$, величина которого возрастает при повышении температуры со скоростью $d\left(\Delta E_{c}\right) / d T \sim 2 \cdot 10^{-4}$ эВ $/ \mathrm{K}$.

\section{Финансирование работы}

Работа выполнена при финансовой поддержке Российского фонда фундаментальных исследований (грант № 20-08-00464).

\section{Конфликт интересов}

Авторы заявляют, что у них нет конфликта интересов.

\section{Список литературы}

[1] Y. Ando. J. Phys. Soc. Jpn., 82, 102001 (2013).

[2] J. Heremans, R. Cava, N. Samarth. Nature Rev. Mater., 2, 17049 (2017)

[3] D.M. Rowe. Thermoelectric harvesting of low-temperature heat. In: Modules, Systems, and Applications in Thermoelectrics, ed. by D.M. Rowe (CRC Press, Boca Raton, FL, 2012) p. 23-1.

[4] Thermoelectrics for Power Generation - A Look at Trends in the Technology, ed. by S. Skipidarov and M. Nikitin (InTechOpen, 2016) p. 572.

[5] A.A. Reijnders, Y. Tian, L.J. Sandilands, G. Pohl, I.D. Kivlichan, S.Y. Frank Zhao, S. Jia, M.E. Charles, R.J. Cava, N. Alidoust, S. Xu, M. Neupane, M.Z. Hasan, X. Wang, S.W. Cheong, K.S. Burch. Phys. Rev. B, 89, 075138 (2014).

[6] I. Mohelsky, I. Dubroka, A. Wyzula, J. Slobodeniuk, A. Martinez, G. Krupko, Y. Piot, B.A. Caha, O. Humlíček, J. Bauer, G. Springholz, M. Orlita. Phys. Rev. B, 102 (8), $085201(2020)$
[7] J. Lee, J. Koo, Y.M. Jhon, J.H. Lee. Opt. Express, 23 (5), 6165 (2015).

[8] R. Sun, S. Yang, X. Yang, E. Vetter, D. Sun, N. Li, L. Su, Yan Li, Yang Li, Z. Gong, Z. Xie, K. Hou, Q. Gul, W. He, X. Zhang, Z. Cheng. Nano Lett., 19, 4420 (2019).

[9] N. Xu, Y. Xu, J. Zhu. npj Quant. Mater., 2, 51 (2017).

[10] T.-H. Liu, J. Zhou, M. Li, Z. Ding, Q. Song, B. Liao, L. Fu, G. Chen. Proc. Natl. Acad. Sci., 115, 879 (2018).

[11] D. Baldomir, D. Failde. Sci. Rep., 9, 6324 (2019).

[12] А.Н. Вейс, Л.Н. Лукьянова, В.А. Кутасов. ФТП, 51, 873 (2017).

[13] Ю.И. Уханов. Оптика полупроводников (Л., Изд-во ЛПИ им. М.И. Калинина, 1970) ч. 1, гл. 2, с. 60.

[14] E. Haga, H. Kimura. J. Phys. Soc. Jpn., 19, 1506 (1964).

[15] H. Köhler. Phys. Status Solidi B, 78 (1), 95 (1976).

[16] А.Н. Вейс, М.К. Житинская, Л.Е. Шелимова. Докл. ХІІІ Межгос. сем. „Термоэлектрики и их применения“ (СПб., 2013) c. 144.

Редактор Г.А. Оганесян

\section{Optical absorption associated with interband and intersubband electron transitions in bismuth telluride}

\author{
A.N. Veis ${ }^{1}$, L.N. Lukyanova ${ }^{2}$, O.A. Usov ${ }^{2}$ \\ ${ }^{1}$ Peter the Great St. Petersburg Polytechnic University, \\ 195251 St. Petersburg, Russia \\ 2 loffe Institute, \\ 194021 St. Petersburg, Russia
}

\begin{abstract}
The spectral dependences of the absorption coefficient $\alpha$ in a submicron samples of the $n-\mathrm{Bi}_{2} \mathrm{Te}_{3}+\mathrm{CdBr}_{2}$ topological insulator, which have optimal thermoelectric properties at high values of the Seebeck coefficient at temperatures below room temperature, are studied at temperatures of 205 and $300 \mathrm{~K}$. The components of optical absorption spectra associated with interband and intersubband electron transitions are revealed and analyzed. It was found that in bismuth telluride, with decreasing temperature, the optical transition of electrons at the interband absortion threshold remain direct and allowed. The values of energy gaps between the absolute extrema of the conduction and valence bands, as well as between the main and additional subbands of conduction band, are estimated. It is shown that the rates of their change with temperature are opposite in sign. The energy band diagram of $\mathrm{Bi}_{2} \mathrm{Te}_{3}$ is constructed, which corresponds to experimental data.
\end{abstract}

